\title{
CMS Workflow Execution Using Intelligent Job Scheduling and Data Access Strategies
}

\author{
Khawar Hasham, Antonio Delgado Peris, Ashiq Anjum, Dave Evans, Stephen Gowdy, José M. Hernández, \\ Eduardo Huedo, Dirk Hufnagel, Frank van Lingen, Richard McClatchey, and Simon Metson
}

\begin{abstract}
Complex scientific workflows can process large amounts of data using thousands of tasks. The turnaround times of these workflows are often affected by various latencies such as the resource discovery, scheduling and data access latencies for the individual workflow processes or actors. Minimizing these latencies will improve the overall execution time of a workflow and thus lead to a more efficient and robust processing environment. In this paper, we propose a pilot job concept that has intelligent data reuse and job execution strategies to minimize the scheduling, queuing, execution and data access latencies. The results have shown that significant improvements in the overall turnaround time of a workflow can be achieved with this approach. The proposed approach has been evaluated, first using the CMS Tier0 data processing workflow, and then simulating the workflows to evaluate its effectiveness in a controlled environment.
\end{abstract}

Index Terms-Data cache, grid, latency, pilot jobs, workflows.

\section{INTRODUCTION}

$\mathbf{S}$ CIENTIFIC experiments such as the CMS experiment [1] at CERN, Geneva, produce large amounts of data, which are then processed by a variety of applications and analyzed by users around the world. Various forms of scientific analyses, data reconstructions and data derivations (called data processing) are performed on the scientific data. These data processing tasks are defined as workflows (collection of tasks and dependencies) associated to a dataset (collection of data files). Each workflow processes thousands of files, executes tasks/steps (known as jobs) and takes care of the dependencies between these tasks. An example of such a workflow is the CMS Tier0 workflow [2], which processes CMS data at CERN.

Manuscript received October 27, 2010; revised February 12, 2011; accepted March 06, 2011. Date of publication May 27, 2011; date of current version June 15, 2011.

K. Hasham and A. Anjum are with UWE Bristol, Computer Science, Bristol, U. K.

A. Delgato Peris and J. M. Hernández are with CIEMAT, Basic Research, Madrid, Spain.

E. Huedo is with the Universidad Complutense de Madrid, Computer Science, Madrid, Spain.

D. Evans and D. Hufnagel are with Fermi National Accelerator Lab. (Fermilab), Computing, Batavia, IL 60510-5011 USA.

S. Gowdy is with CERN Geneva, CMS, Geneva, Switzerland.

F. van Lingen is with Radio Television Suisse, Multimedia R\&D, Geneva, Switzerland.

R. McClatchey is with UWE, Bristol, CCS Research Centre, CEMS faculty, UWE, Coldharbour Lane, Frenchay, Bristol, Avon BS16 1QY, U.K.

S. Metson is with H. H. Wills Physics Laboratory-University of Bristol, Physics, Bristol, UK.

Color versions of one or more of the figures in this paper are available online at http://ieeexplore.ieee.org.

Digital Object Identifier 10.1109/TNS.2011.2146276

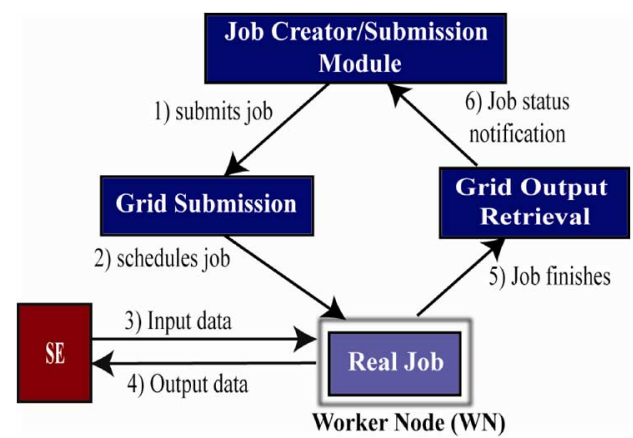

Fig. 1. Job life cycle in a grid environment.

The turnaround time of these workflows depends upon the number of files being processed, the number and nature of the tasks within the workflow and the level of parallelism that can be achieved. Parallelism is achieved if a task execution can be split into multiple jobs. A job is a workflow task that runs on a given data.

The turnaround time of a workflow, running on a single machine, is simply the sum of the execution times of individual tasks in that workflow. It would take an enormous amount of time to execute a complete workflow on a single machine due to the size of the datasets used within high energy physics. The problem is further compounded if a number of users submit multiple data processing jobs, each in turn consuming multiple datasets, in order to achieve desired results. Workflow jobs, whose requirements have been met and have no dependencies, can run in parallel in a distributed environment. Therefore, tasks in scientific workflows are preferably executed on distributed resources to reduce the overall execution time and to enable users to achieve rapid throughput.

A highly distributed environment such as the Worldwide LHC Computing Grid (WLCG) [3] has been deployed for the analysis of data from the Large Hadron Collider (LHC). In this distributed environment each workflow job would face scheduling and data access latencies during its lifecycle (see Fig. 1). The WLCG is a global collaboration of more than 170 computing centers in 34 countries that combines the computing resources of more than 100000 processors. The mission of the WLCG project is to build and maintain data storage and an analysis infrastructure for the entire high energy physics community that will use the data from the LHC at CERN. At full operation intensity, the LHC will produce roughly 15 petabytes (15 million gigabytes) of data annually that thousands of scientists around the world will access and analyze. 


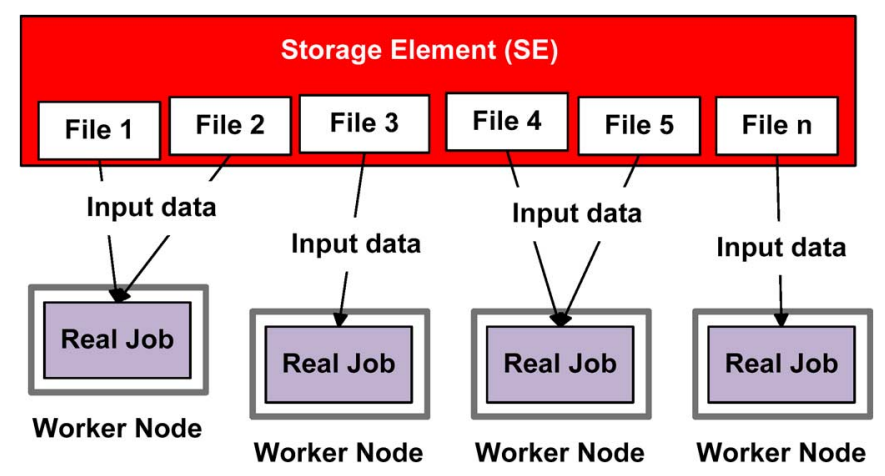

Fig. 2. Multiple jobs accessing an SE.

Grid scheduling latency is the cumulative time spent in discovering resources in a Grid for scheduling and the waiting time that is spent in the queues of meta and local schedulers before a job can start execution on a so-called worker node (WN). A worker node is an execution resource at a site. Here the data access latencies are mainly caused by the network bandwidth limitations, the load on a storage element (SE) and the time spent in accessing a storage media such as a tape drive [4]. These latencies can affect the turnaround time of the workflow and in some cases can exceed the overall execution time of a job. An experimental study [5] has shown that it takes almost five minutes (on average) for a job, in the EGEE Grid [6], to start its execution from the time it was submitted. One can understand the extent of delays if there are thousands of jobs being submitted and executed in a Grid infrastructure such as the WLCG. Minimizing these latencies is a major research challenge in order to offer a high quality of service that users expect from production Grids.

With the current data storage hierarchy of the WLCG, each site maintains one or multiple dedicated machines called SEs to store data. Each job can access the data from a given SE. The jobs in the CMS Tier0 workflow (detailed in Section III) stream data directly from chunks of the data available on the SE. These jobs process this data, without downloading the entire dataset on the local hard disk of a worker node. This mechanism (see Fig. 2) creates an additional burden on the SE if every CPU-bound job remotely accesses small chunks of the data periodically leading to a high frequency of $\mathrm{I} / \mathrm{O}$ requests. An SE has to keep the files open, as they are being read, for longer periods of time and this can add to the latency being faced by the other data requests.

Storage systems such as CASTOR [7] can store petabytes of data; however, such systems are vulnerable to performance issues in terms of high access latencies and this becomes worse with increasing loads. This leads to longer data access times and thus affects the overall execution time of the workflow. In order to reduce these data access and scheduling latencies and to improve the workflow turnaround time, this paper proposes to use a pull-based scheduling system and to establish data caches on the worker nodes. This can be achieved by managing the resources of a worker node by using a customized resource management software component.

To demonstrate this work, the proposed approach makes use of a global scheduler and the concept of a pilot job. A pilot job is a job that is responsible for setting up the required execution environment and for managing the execution of a real job. A real job is a job that is part of a user workflow and that waits in the global scheduler queue. In this paper, the term real job and job will be used interchangeably. Both these jobs follow different submission and scheduling mechanisms. A pilot job follows the traditional grid submission mechanism; however, a job will bypass it because a pilot job downloads it from a global scheduler queue for execution. With the help of this approach, a pilot job can assist the job in finding all or some of its required files in the cache maintained on the worker nodes. A job can start its execution as soon as it has been scheduled to a pilot job, thus reducing the queuing and scheduling delays. The job will first look for its input files in the cache and will read the data from the local cache, provided the cache holds the required data; otherwise, the job will contact the given SE for the data. Once a job has completed its execution, a pilot job immediately notifies its completion status to the scheduling and monitoring components, thus minimizing the delays that otherwise exist in retrieving and notifying the job completion status. This approach is further explained in Section IV.

This paper is organized as follows. Section II discusses the state of the art in the research domain. Section III briefly outlines the Tier0 workflow specification and execution system being used at CMS-CERN. This workflow is being taken as a case study to demonstrate that the proposed approach is effective in improving the data access, queuing, scheduling and execution latencies in real scientific computing environments. Section IV provides details of the proposed architecture and justifies its selection in solving the problem. Section $\mathrm{V}$ provides a description of the results which show that the proposed solution has been quite effective in reducing the turnaround times of large workflows. Section VI concludes this paper with possible directions for future work.

\section{RELATED WORK}

Numerous efforts have been made to reduce data access latencies in intensive data processing applications. The replica management service [8] of the Globus toolkit uses data replication in order to optimize the performance of a data analysis process. The data replication is done at the site level; however, it cannot solve the latency issues resulting from a large number of open file pointers on the SE and a large number of I/O requests. Intelligent Cache Management (ICM) [9] uses the concept of a local data cache to optimize query performance but it replicates and stores the data on a regional basis. None of these approaches exploits the resources at worker nodes for the purpose of data caching. Peer-to-peer (P2P) approaches [10] have been using end node capabilities for data storage; most notably, BitTorrent [11] and super-peer approaches such as KaZaa [12] use end node capabilities for data discovery and data transfer. The BitTorrent approach works on the so-called fair share basis. Data providers have to supply data for consumption by consumers in the outside world, which puts additional burden on the network usage and could also be against the security policies of the Grid sites. Taylor [13] proposes a framework that uses the concept of super peers to create an application-specific or workflow-specific data cache overlay. This approach makes 
use of AlChemist's built-in flexibility to support a P2P infrastructure on top of the WSPeer API for communication with its peers. However, this approach is dependent upon the AlChemist framework and the WSPeer API to create data cache overlays on dedicated data nodes, whereas we have proposed to create data cache on every worker node inside a cluster to optimally use the available resources in the Grid infrastructures. There have been various approaches [14] to schedule job taking into account the location of the data needed by a job to minimize the data transfer, and thus the job execution time.

In addition to these efforts, research has been carried out to minimize job submission and output retrieval latencies by using the concept of pilot jobs in Grids. Grid projects such as PanDA [15], DiRAC [16] and AliEn (Alice Environment) [17] use this approach to schedule and execute jobs. All these projects use the pilot jobs to reduce the job submission latency by pulling a job from a global job queue and thus provide a fault-tolerant job execution mechanism. However, these systems do not use a pilot job infrastructure to reduce the data access latencies. A project in CMS, GlideInWMS [18], makes use of grid resources as part of its Condor pool. It uses Condor [19] glidein which acts as a pilot job on a worker node. It takes the leverage of the Condor infrastructure to enable communication with different Condor daemons. Since these glideins are often running behind a firewall, it uses a workaround called Condor's Generic Connection Brokering (GCB) [20] which helps the global scheduling daemons to contact these glideins and to push the actual jobs directly to them. However, this approach has led to scalability problems [21]. Moreover, it does not support the data cache mechanism on worker nodes to reduce data access latencies.

The work done by Shankar et al. [22] is closely related to the work being reported in this paper. Their approach makes use of a dedicated cache space on the worker nodes in an execution cluster for the data caching purpose. They accomplish this with the help of condor-DAGMan, which makes it specific to the Condor environment only. Its scheduling process involves prior planning of the resources for a given DAG; however, in environments such as CMS, jobs are generally data driven and are not completely known until they have been created. Moreover, the scheduling is performed within a single site and hence is not suitable for heterogeneous environments like the WLCG Grid.

\section{CASE Study}

The CMS experiment at CERN uses a multi-tier distributed architecture [23] where CERN is the Tier0. Using a four-tiered architecture (from CERN's central computer as the Tier0 to small Tier3 analysis clusters), CERN distributes LHC data and computations across resources worldwide to achieve aggregate computational power unprecedented in high energy physics data analysis research. The Tier0 reformats, writes out primary datasets, stores this raw data, generated from the output of the CMS Detector, performs an initial data reconstruction and distributes the processed data to Tier1s. In data reconstruction phase, the raw data from the detector is converted to FEVT [2] format that is then used in analysis by the physicist. In this

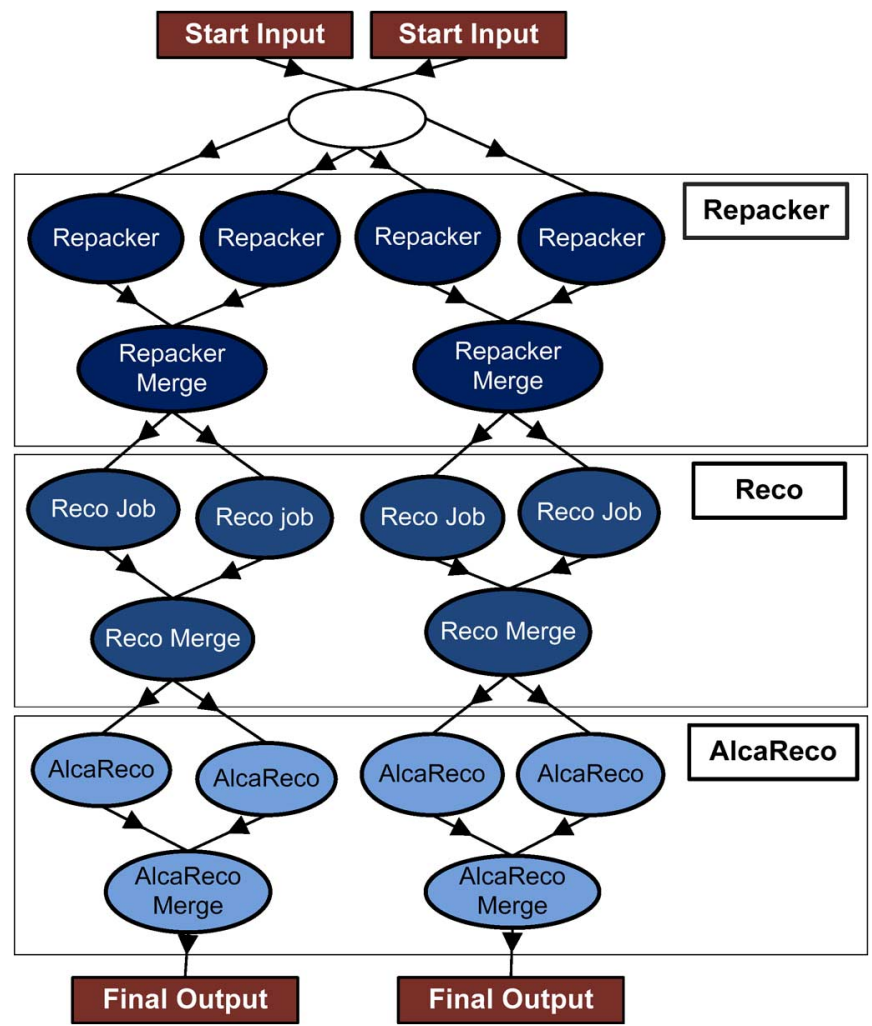

Fig. 3. Tier0 workflow for CMS at CERN.

paper we concentrate on the Tier0 workflows and their data access patterns; however, the approach being discussed in this paper should be of wider usability, especially for other CMS data intensive workflows that we intend to demonstrate in the future. For the initial data reconstruction, a Tier0 workflow is used, which is also a sample workflow to evaluate and benchmark the proposed system. This workflow consists of three main steps: 1) Repacker, 2) PromptReco and 3) AlcaReco. The Repacker jobs reformat the binary data from CMS detector and split the output into different primary datasets based on physics information. The PromptReco jobs take this output as their input and perform an initial reconstruction into usable sets of physics data such as the particle trajectories and the properties of the candidate particles. The AlcaReco jobs perform a much higher selectivity of the data produced by the PromptReco jobs and also carry out some processing on this smaller subset. This output is used to align and calibrate the CMS detector. Fig. 3 shows the CMS Tier0 workflow.

In each step, several jobs are created. The number of jobs in each step depends on the number of physics events (or filtered particle collisions of interest) in the input files. A physics event in the CMS experiment is a collision of protons that gives birth to elementary particles [24]. Currently each job has to process around 5000 CMS physics events. Each job produces a relatively small output dataset compared to its input dataset. It is inefficient to store and transfer smaller files to a tape-based central storage system because the process encounters delays and latencies in transferring a file to and from the tape drives. Therefore, each step has a special job called the Merge job, which gets the output from multiple jobs and merges them. Only the merged 
files should, ideally, be transferred to the central storage system in the first instance.

The creation and execution of all the workflow steps is data driven. The workflow starts execution whenever a new file is available that requires some kind of processing. The unnamed oval process in Fig. 3 triggers the first step by creating the Repacker jobs. The subsequent jobs are created according to the system policies, workflow rules and data availability. There are two main characteristics of this workflow. Multiple jobs are dependent on a single input file, and a single job, which is the Merge job, is dependent on multiple smaller files produced by earlier jobs in the workflow. This workflow is created and executed by a ProdAgent [25]: a workflow management system used in CMS. ProdAgent is a component-based system driven by an asynchronous and persistent messaging system for communication among these components. ProdAgent is responsible for creating, submitting and then monitoring the jobs in a CMS workflow. In the existing setup, all jobs within a Tier0 workflow are queued up in the global scheduler of ProdAgent. The global scheduler can schedule the jobs on the available sites in the Grid using the configured submission mechanism such as gLite [26] and Condor-G [27]. The Tier0 instance uses local LSF [28] submission.

Once a job has been scheduled by the meta-scheduler, it is sent to a local scheduler such as LSF, PBS [29] or Condor which runs on a particular site. A job has to wait in the local scheduler's queue before it is scheduled to a worker node. After arriving on the worker node, the data dependent jobs have to wait again before their required datasets come online on the given SE for streaming. Once the job can access the data, it reads data in chunks and performs its processing. After completing the processing of its data, the job stages back the output to a given SE. It then faces further delays until a monitoring component knows that a job has been finished and it has staged back its output. The latency in retrieving the job completion information delays the submission of a dependent job, thus increasing the workflow turnaround time. In the current execution environment, as shown earlier in Fig. 1, each job has to face the scheduling, monitoring and data access latencies. These latencies affect the execution time of an individual job, which, in turn, affects the turnaround time of the whole workflow. Moreover, the Tier0 operations have revealed that the data access latencies are severely affected by an overloaded SE.

The CMS Tier0 is a latency critical system, where disk buffers fill up if data from the detector are not processed in a timely manner, and calibration constants derived promptly are used to reconstruct the new data. For instance, data in an Express stream [2] is expected to be processed within $\sim 1-2$ hours. Therefore, reducing aforementioned latencies is very important to improving the turnaround time of the workflow.

\section{Proposed Architecture}

In order to optimize the execution of the CMS data processing workflow, we propose to use a pull-paradigm driven by pilot jobs and to establish data caches on the execution resources. This approach will help in avoiding scheduling, monitoring and data access latencies for the real jobs. As a consequence of this

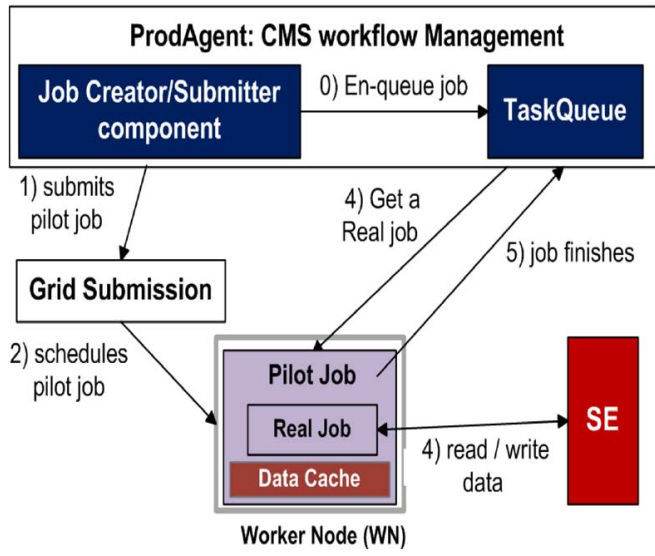

Fig. 4. Overview of the proposed architecture.

approach, there will be fewer job failures that may appear due to incorrect job execution environments. The approach will also be used to create data caches on the worker nodes.

The pilot job concept provides three main advantages. Firstly, jobs do not face scheduling and monitoring latencies since the pilot jobs will pull them directly from the global scheduling queue and notify job completion as soon as a job has been finished. Secondly, the pilot job will manage the available resources on the worker node for data caching, which will help in avoiding data access latencies. Thirdly, the pilot jobs will ensure that an execution environment is appropriate for a job before executing it. Furthermore, the pilot jobs act as a layer on top of the local batch system such as Condor and LSF and therefore it ignores the local schedulers and makes use of the meta-scheduler policies for making scheduling decisions. This not only reduces the queuing times that can be quite high in local schedulers, but it will also reduce the job failures. As a result of this, jobs are only sent to a site if they are requested by a pilot job running on the site and it has the required execution environment. Moreover, this approach makes the decision making process distributed, cooperative and fault tolerant. With this approach, there will potentially be a single scheduler in the Grid for the jobs since they will bypass the local schedulers running on the sites. The meta-scheduler in association with the pilot jobs will make cooperative scheduling decisions to reduce job failures and minimize queuing and execution latencies. This proposed approach dynamically matches jobs to the pilot jobs and thus makes the scheduling decisions that are required for efficient cache and resource usage. The overview of the proposed architecture is shown in Fig. 4.

The JobCreator component of the ProdAgent system will create the jobs from the workflow and queue them in the TaskQueue (TQ). The TaskQueue, a central job queue for ProdAgent jobs, will hold all the jobs of the workflow that are waiting to be scheduled for execution. The TaskQueue will schedule them upon receiving job requests from the pilot jobs. The TaskQueue is also responsible for registering new pilot jobs and maintaining the information about them. An architecture of the pilot job and the TaskQueue is given in Fig. 5.

The number of pilot jobs that should be submitted to a site is subject to the number of jobs that are waiting in the TaskQueue 


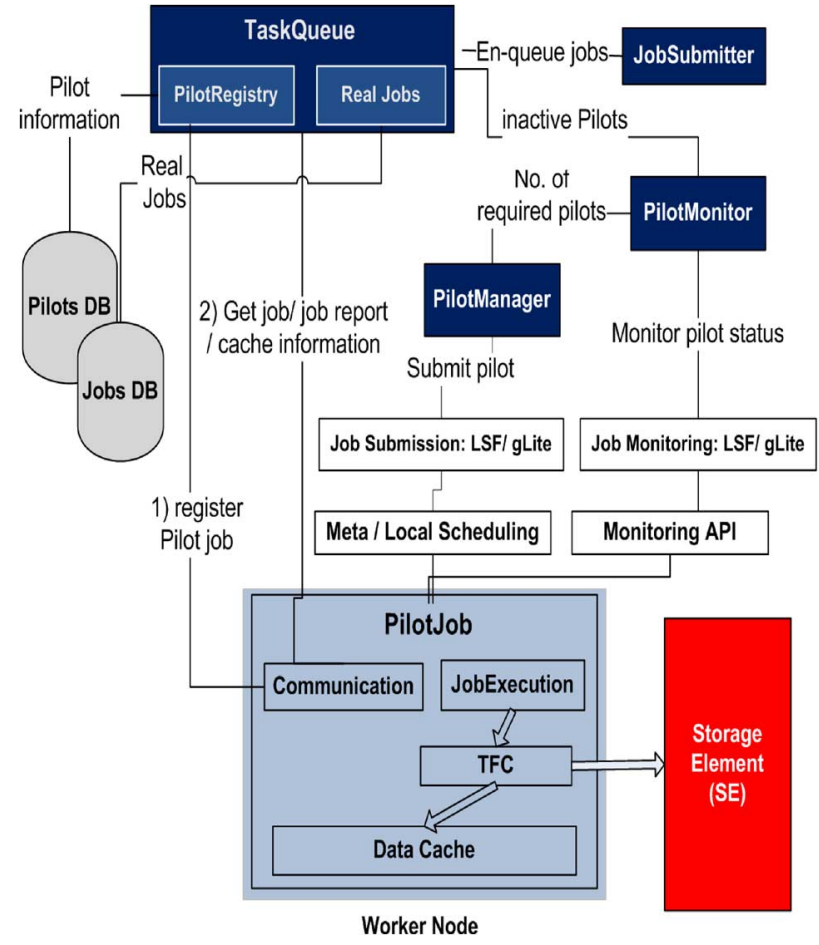

Fig. 5. Detailed architecture of PilotJob and TaskQueue.

for that particular site. Currently, each pilot job is capable of running a single job at any point in time. Since the Grid sites are shared among multiple Virtual Organizations (VOs), we cannot load them with pilot jobs that will not have work to do. Two configurable thresholds are used to avoid this problem. These thresholds are called minPilots and maxPilots, which put a limit on the minimum and maximum number of pilot jobs for a site. Each site has its own values for these thresholds that are provided by the site policy. The PilotMonitor component, which is responsible for monitoring the state of submitted pilots, calculates the required number of the additional pilot jobs within these thresholds and then requests the PilotManager component to submit them. Section IV-A details the algorithm used in the PilotMonitor to calculate the required number of pilot jobs.

Upon receiving the request from the PilotMonitor, the PilotManager component prepares the required number of pilot jobs with configurable parameters and submits them. The pilot jobs are submitted using the underlying submission system such as LSF, Condor or gLite for grid submission. Once a pilot job has been scheduled on a worker node within an execution cluster, it will perform initial environment checks and register itself with the TaskQuеue. In the registration phase, the TaskQueue assigns a unique id, PilotID, to each pilot job to identify it during its subsequent requests. Once the environment has been setup and the registration process has been completed, the pilot job is then ready to contact the TaskQueue to get the job. However, if there is something missing in the environment that is required for executing a job, the pilot job announces the error and terminates itself; hence no job would be executed. This helps in having fewer job failures that occur due to an improper execution environment which is one of the major reasons for job failures in
Grids [30]. The pilot job approach being proposed in this paper will help in reducing such failures.

After the successful environment check, the pilot job contacts a given TaskQueue URL and requests for a job. Section V gives a brief account of the cache-aware scheduling that the TaskQueue performs upon receiving the pilot job request. Once a job has started its execution, it looks for the physical location of its input files. The pilot job maintains a mapping file called Trivial File Catalog (TFC) to discover the input files. This is an XML file which maintains the rules to convert a Logical File Name (LFN) into a Physical File Name (PFN) to locate a file. The TFC first looks into the pilot job's cache area for the required files. It provides a pointer to the input file residing on an $\mathrm{SE}$ if the required file is not available in the cache.

The pilot job mechanism using the job pull-paradigm does not pose security concerns for the grid resources. Sites are normally reluctant to open ports to allow the outside world to make connections with their internal resources. The pilot jobs act as clients for the TaskQueue and pulls jobs into the site preventing opening any additional ports.

\section{A. PilotMonitor Algorithm}

The PilotMonitor component keeps track of the submitted pilot jobs and the jobs that are queued in the TaskQueue. The pilot jobs that are submitted to a site can be in one of three states (inactive, idle, busy) during their lifecycles. The inactive state is applied to those pilot jobs which are not running and have been waiting in the site scheduler. A pilot job will be monitored as idle if it is running on a $\mathrm{WN}$ but could not get a job from the TaskQueue. A busy pilot job means that it has acquired a job and this is in execution.

The PilotMonitor algorithm uses three important thresholds to calculate the required number of pilot jobs for a site. These thresholds are the maximum and minimum number of pilots to be submitted to a site and the minimum number of idle pilots. These thresholds are represented as minPilots, maxPilots, and minIdlePilots, respectively. This algorithm makes sure that the required number of the pilot jobs should not exceed the $\operatorname{maxP} i$ lots threshold and also they should not be less than minPilots. The last threshold, minIdlePilots, may be useful for sites like Tier0, where it may be desirable to always keep some idle pilots that are ready to accept a job. This minimizes the delay caused by the pilot job submission and also reduces the submission time for the jobs that are submitted for the first time. All these thresholds are configurable, according to the site policy.

The PilotMonitor runs this algorithm periodically for every known site in its list. The algorithm is summarized as follows.

\section{PilotMonitor algorithm}

1. Recall thresholds and previously submitted pilots for site

2. Set: available slots $=$ maxPilots threshold submitted pilots

3. If (available slots $<=0$ )

4. Then: Do not continue (do not submit more pilots) 
5. Query TaskQueue about tasks that can run on this site

6. For each group of enqueued tasks:

7. If (enqueued tasks $<$ inactive pilots)

8. Then: mark inactive pilots as active, mark tasks as covered

9. Else: If (available slots $>$ number of tasks)

10. Then: send more pilots, mark tasks as covered

11. if (idle pilots $<$ minIdlePilots):

12. Then: send more pilots

13. if (submitted pilots $<$ minPilots):

14. Then: send more pilots

This calculation is then passed to the PilotManager component which submits the pilot jobs to a given site.

\section{B. Cache Replacement Algorithms}

On a worker node, each pilot job will have limited space available for caching so an efficient caching replacement algorithm is required for managing the cache on worker nodes. There are many caching algorithms [31] that can perform this task including the traditional algorithms such as First-In-First-Out (FIFO), Least Recently Used (LRU), and Least Frequently Used (LFU). The traditional algorithms offer low overhead as they need minimal information, such as reference count and last access time, for their cache replacement policies. Here reference count means the number of times a file has been accessed in the past and the last access time means the time at which a file was last accessed. Some improvements have been made in these classical algorithms namely LFU-*, LFU-again, LRU-K [32], and LCB-K [33]. These improved algorithms such as LFU-* remove the cache pollution problem faced by LFU. The LCB-K and other cost sensitive cache algorithms [31] consider the cost of data removal from the cache. However, these improved algorithms store extra information to deal with issues that occur with the traditional algorithms.

The nature of the CMS Tier0 workflow favors the LRU algorithm because once a step has been completed and its output has been merged, the smaller files are no longer required in the following steps. These smaller files are only required by the jobs that were generated at the same level in the workflow hierarchy. The jobs in the following steps use the data from the merged output that has been produced from the smaller files in a previous step. Moreover, the jobs in the CMS workflow do not directly interact with the pilot job's caching component for a cache lookup because they use the TFC to locate the physical location of a file. For these reasons, it is somewhat difficult for the cache component to maintain a reference time history or the reference count, used by the LFU, for each file in its cache. Consequently, for our prototype implementation, we have used LRU because of its simplicity and its compatibility with the CMS workflows.

\section{Data Caching Policy}

A pilot job running on a worker node can control resources for the time it is allowed to run. Each job, running within the pilot job, will consume some input files and generate some output files. Apart from executing a job, the other important task of a pilot job is to maintain these data files in its cache. The caching policy must adhere to the requirements and constraints detailed in the following paragraphs.

Each running pilot job will be given a certain amount of disk space. The pilot job uses this space to download jobs and maintain output files. This space will become the pilot job's cache area. This space is configurable at the site level and this is decided by the site administrator. In CMS, each job is given a 10 GB space on the disk. Each pilot job will also get at least 10 GB space that acts as the maximum allowed space for the data storage. Since the jobs are executed within the pilot job space, as shown in Fig. 5, we will always need a minimum space available at any given time. This minimum space is used by the real job to temporarily store its output that has been produced from the job execution. Let us call this required minimum space a Min $_{\text {Threshold. }}$ The total space that can be utilized for caching data can be given as

$$
\text { Cache }_{\text {Size }}=\text { Max }_{\text {Space }}-\text { Min }_{\text {Threshold }} \text {. }
$$

This ensures that we always use the maximum allowed space for caching purpose by always keeping the minimum available space for the job execution.

Let us say we have a set F of "n" cache files $\{f 1, f 2, f 3 \ldots f n\}$, each having the sizes $\{\mathrm{S} 1, \mathrm{~S} 2, \mathrm{~S} 3 \ldots \mathrm{Sn}\}$, respectively, such that their collective sum is less than or equal to cache size

$$
\sum_{i=1}^{n} S i \leq \text { Cache }_{\text {Size }} .
$$

For example, a job produces a new file $\mathrm{X}$ which is required to be placed in the data cache. The file $X$ would become part of the cache if the required space is available. If the remaining space in the cache is insufficient to accommodate this new file, then we need to remove some files from the cache. The LRU algorithm should remove files from the cache such that the sum of the removed files matches the following criterion:

$$
\sum_{i=1}^{m} S i \geq \operatorname{RequiredSize}
$$

where RequiredSize is the size of the new file for which the cache replacement algorithm will create space in the cache.

\section{Cache Sharing Among Pilots on Same Worker Node}

On execution if resources are available at Tier0 at CERN, multiple jobs can run in parallel on a single WorkerNode (WN). Therefore, it is possible that multiple pilot jobs may land on the same WN. The usage of the cached data will become more effective if these pilot jobs can share their cached data. Since the cached data is available and accessible locally, there will be low data access latencies if the jobs can access the shared cache. The cache sharing concept becomes even more helpful in the scenario when Pilot1 is running a job which needs a file 
available in the cache of Pilot 2 that is running on the same WN. A job does not need to access an SE if pilots can locate and then share this cached data. This will also increase the cache hit rate.

We propose an approach that is called cache-per-host to establish cache sharing among the pilot jobs running on the same WN. Here we assume that the pilot jobs share the same file system on a WN. In the case of CMS, all the pilot jobs run under the same user id or the users belong to the same group; therefore, they can access each other's directories. When a pilot job arrives on a WN and registers itself with the TaskQueue, the TaskQueue sends back the list of other available pilot jobs on that WN and their cache locations in response to this register request. The pilot job will then save this list and poll the given locations for new cache files. A Unix hard link to a newly found file is created into the pilot's own cache area and the file is placed into the cache by using the LRU algorithm that has been discussed previously. In this way, the file remains in the system even if the original owner of that file deletes it. A file is removed from the system only if its last link is deleted. At this point, a pilot job that prompted the file delete operation will notify the TaskQueue about this. In cache-per-host, the total space available to a pilot job on a worker node for data caching is dynamic. It is calculated as a function of the number of pilot jobs on that worker node, the maximum space allowed to each pilot job and the $\operatorname{Min}_{\text {Threshold }}$. The following equation shows this model where num_pilotjobs is the number of pilot jobs on that worker node:

$$
\text { Cache }_{\text {Size }}=\text { Max }_{\text {Space }} \times \text { num_pilotjobs }- \text { Min }_{\text {Threshold }} \text {. }
$$

Since the pilots can shut themselves down or new pilots can arrive on the same WN at random, a mechanism is required to update the running pilot jobs about the other available pilot jobs on a particular WN. This is achieved by making use of the "Heartbeat" message, which a pilot job regularly exchanges with the TaskQueue. This message informs the TaskQueue that a pilot job is alive. In response, the TaskQueue provides the pilot with an updated list of other pilot jobs on the same WN. In this way, each pilot job updates itself about every other pilot job running on the same WN. When a pilot job polls the given pilot jobs' locations if that location is not accessible, then the pilot job removes that entry from its list and that particular pilot job is assumed to be dead. Each pilot job will update its list of the pilot jobs after each "Heartbeat" message.

\section{E. Cache-Aware Task Scheduling}

Each job placed in the TaskQueue provides its requirements, such as its preferred site and input files. When a pilot job submitted to a worker node starts execution, it will contact the TaskQueue to get a job that meets its requirements. The request to the TaskQueue includes its PilotID, Host, SE, Time-to-Live (TTL) and cached files. In this request, PilotID is the id assigned to each pilot job during its registration with the TaskQueue, Host is the name of the worker node where the pilot job is running, SE is the name of the storage element accessible to the pilot job in that particular site and the cached files are the files available in the pilot job's cache. The TTL is the remaining life of a pilot job. In the current implementation for the Tier0, the pilot jobs can run forever because resources are dedicated to Tier0 operations. But this information will be configurable in future implementations and will be added into the job scheduling process.

The TaskQueue performs the job scheduling by comparing job requirements against the pilot job information. The scheduling algorithm must schedule a job to a pilot job whose maximum requirements meet the information provided by the pilot job. The caching information is used to match job data dependencies against the files maintained by the pilot job. The TaskQueue schedules a job to a pilot job that has the maximum number of jobs required files in its cache. A job, arriving on a pilot job that holds some of the required files in its cache, will face less data latency since it can find some or all of its required files in the pilot job's cache. The job without any specific requirement can be scheduled onto any pilot job.

In order to provide improved job scheduling and to use cache more effectively, we implemented a waitForData policy alongside the above-mentioned scheduling model. According to this policy, when a pilot requests a job but cannot match the data dependencies of a job, the TaskQueue would not schedule the job to the pilot if there are other idle pilots holding the required data. The TaskQueue would wait for these idle pilots to eventually request a job. In this way, the scheduling process encourages the maximum number of reads from the cache. However, if there are no other pilots that hold the required data or they are not idle, the TaskQueue will schedule the job to a pilot that does not have the required files instead of keeping the job for an unknown period of time, because, as a last resort, a job can always access data from an SE.

\section{EXPERIMENTAL RESULTS}

A series of experiments have been conducted at CERN's Tier0 infrastructure. For these experiments, a test bed has been used that comprises a cluster of ten machines, each of which is capable of running four jobs in parallel. We used a dedicated resource in Tier0 as an SE to avoid any external influence on the SE. The CMS Tier0 reconstruction workflow is used as a sample workflow in these experiments. This workflow generates a total of 172 jobs, requires 83.41 Giga bytes (GB) of input data, and produces $112 \mathrm{~GB}$ of output data. Several iterations of the complete Tier0 workflow have been executed with the existing system, i.e., ProdAgent and with the new developed prototype based on pilot jobs and cache. These experiments have been repeated several times. The figures show the measured average values and the error bars represent the standard deviation of the mean. The results in Fig. 6 show that the workflow turnaround time has been reduced by $4 \%$ when using the proposed system.

This reduction in the turnaround time is mainly due to the reduction in job submission and job status notification times since the pilot-based approach reduces the job scheduling latencies (explained in the discussion of Fig. 7). The reduction is small because, given the size of the test-bed, most of the jobs had to be queued in LSF, and thus the job submission delays were not reduced. Moreover, in these tests, it was not possible to measure the behavior of the proposed system against different parameters such as job failure rates, queuing times and data access latencies. This is mainly due to the fact that there was no additional load 


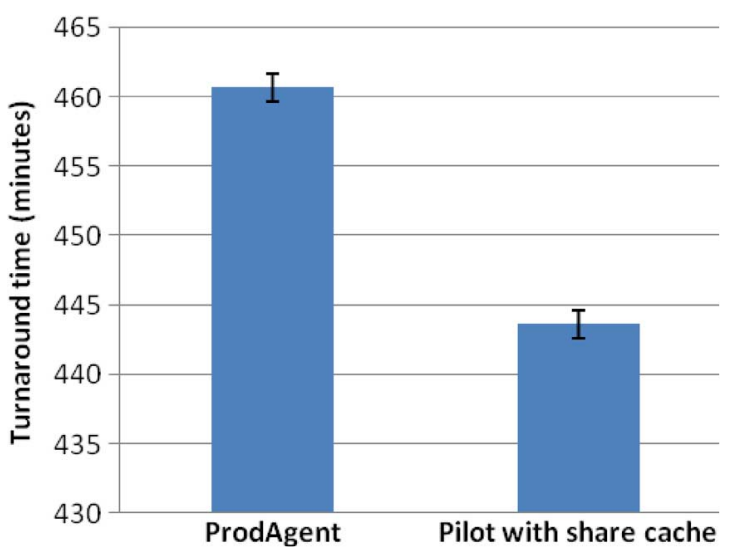

Fig. 6. Effect of pilot-based system on the Tier0 workflow turnaround time.

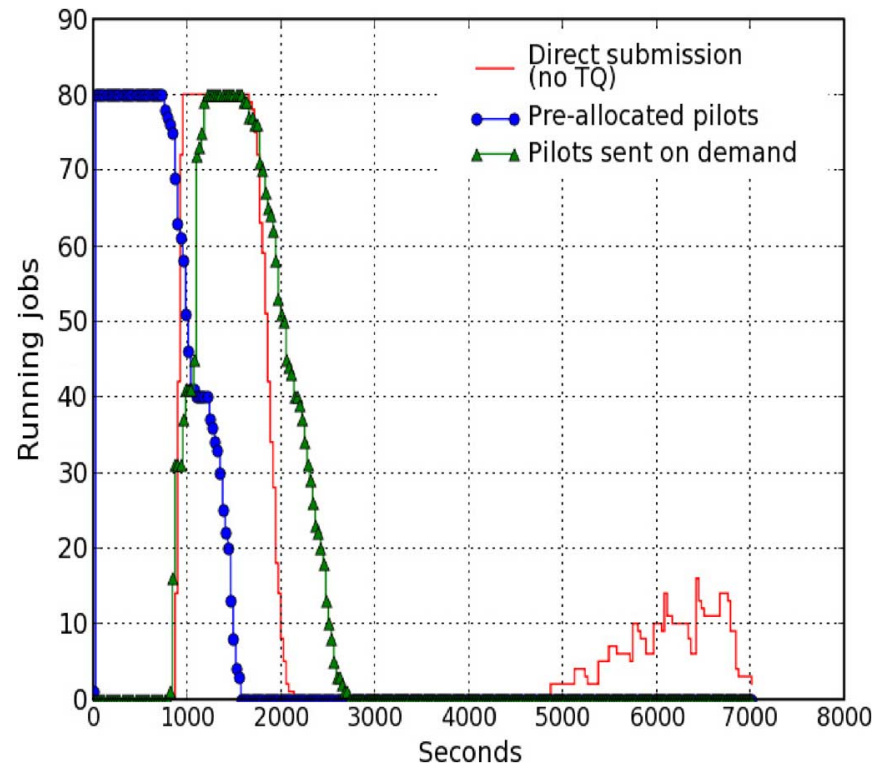

Fig. 7. Number of jobs from W3 workflow submitted and running over time using different submission systems.

on the SE as it is only being used for data access operations in these experiments.

It was not practically feasible to artificially alter the access conditions on the SE that has been used in the Tier0 experiments. Therefore, a variety of simulation experiments were conducted at CIEMAT (in Madrid, Spain), which is a CMS Tier2 site, to evaluate the impact of the pilot jobs and their data caching patterns. In these experiments, 120 con-current pilot jobs were executed. This number is not far (i.e., the same order of magnitude) from the number of pilots a production CMS site would run. Also note that for the case of multi-site setups, the cache of the pilot jobs at each site behave independently of the others because there are no inter-site job dependencies.

For the simulated experiments, a simulation engine has been implemented to emulate the ProdAgent and the data driven behavior of CMS workflows by using a concept called "steps". A workflow is divided in such a way that jobs in the next step depend on the output produced in a previous step. Three types of workflows, generating the jobs in two steps, i.e., step0 and stepl, have been used in these experiments. These three types of workflows display three different characteristics of data intensive scientific workflows in general and the CMS Tier0 workflow in particular. As mentioned in Section IV, the jobs can display various types of data dependencies. It can be a one-to-one (serial chain) dependency, or many-to-one dependency where one job (the merge job) consumes the files produced by two or more jobs in the previous step, or one-to-many dependency where multiple jobs (splitting jobs) can consume the files produced by a single job.

The serial chain workflow (abbreviated as W1) demonstrates a one-to-one dependency. In this workflow (W1), 80 jobs that produce 80 files as their outputs are created in step0. This is followed by another 80 jobs in step1 that are dependent on the output produced in step0. This workflow represents a one-to-one dependency between the jobs in the workflow. In the second workflow (W2), 40 jobs are created in step0 that produce 40 files and are followed by 80 jobs in step1. In the second workflow, two jobs in step 1 are dependent on a single file produced by a job in step0. This workflow represents a splitting workflow where more jobs consume the data that has been produced by fewer jobs in the previous steps. In the third workflow (W3), 80 jobs in step 0 produce 80 files and are followed by 40 jobs in step1. This is an example of a merging workflow where two or more than two jobs are merged in the subsequent steps of a workflow. Each job in these workflows produces a file of size 700 Mega bytes (MB). In each workflow, the jobs in step0 are first generated and scheduled, and then the jobs in step1, which depend on the data produced by the jobs in step 0 , are generated and enqueued in the TaskQueue.

In order to study the effect of the proposed approach on different type of workflows under different SE conditions (given in Table I), two different parameters, the delay factor and the failure rate, are used for these experiments. The delay factor is a delay that a job bears in accessing an SE. It is used to simulate the delays, which occur due to the load on an SE, in reading and writing processes. A higher delay factor means longer times are being taken in reading and writing to the SE. The values for delay factor used for these simulations are $0.01,0.15$, and 0.50 and are represented as d1, d2, and d3, respectively. Each individual job may experience slightly different delays due to multiple uncontrollable factors such as the exact time when each job reads or the latency caused by a disk server in which each of the accessed files is located. We have modeled this by randomly selecting job delays from a Gaussian distribution using the selected delay factor for the workflow. The other factor, failure rate, is used to simulate the probability of failure in reading or writing data to an SE which eventually means failure of a job; hence, it may have a negative effect on the workflow execution. The values for failure rate used are $0,0.03$, and 0.1 and are represented as f1, f2, and f3, respectively. A higher failure rate means higher chances of failure in reading and writing data from and to a data source.

Different combinations of these two factors give us different load conditions on an SE. The d1f1, d2f2, and d3f3 combinations represent Low, Moderate, and High loads on an SE, respectively. The Low load on an SE means that there are not too many read and write requests to the SE; therefore, jobs would 
TABLE I

COMBINATION OF DELAYS AND FAILURE FACTORS

\begin{tabular}{|l|l|l|l|}
\hline Combination & $\begin{array}{l}\text { Delay } \\
\text { factor }\end{array}$ & $\begin{array}{l}\text { Failure } \\
\text { factor }\end{array}$ & $\begin{array}{l}\text { Load on SE } / \text { SE } \\
\text { condition }\end{array}$ \\
\hline $\mathrm{d} 1 \mathrm{f} 1$ & $\mathrm{~d} 1=0.01$ & $\mathrm{fl}=0$ & Low/Normal \\
\hline $\mathrm{d} 2 \mathrm{f} 2$ & $\mathrm{~d} 2=0.15$ & $\mathrm{f} 2=0.03$ & Moderate/Medium \\
\hline $\mathrm{d} 3 \mathrm{f} 3$ & $\mathrm{~d} 3=0.50$ & $\mathrm{f} 3=0.1$ & High/Worse \\
\hline
\end{tabular}

not face long data access delays. The Moderate load on an SE means that there are a reasonable number of read and write requests to the SE and jobs might face slight delays in reading or writing files. The High load means that there are a huge number of requests pending for reading and writing the data to the SE; consequently, the jobs will face longer delays and a higher probability of failure. Table I summarizes these combinations.

A third factor that can influence the experiments is the caching scheme used in an experiment. The effect of the data caching on such environments (such as in CMS) is more prominent since this can significantly influence the overall execution time. Different cache schemes such as cache-per-host (C1), single-pilot-cache $(\mathrm{C} 2)$ and cache-per-host without waitForData logic (C3) have been used in these experiments. In the single-pilot-cache, the pilot jobs running on a WN do not share their cache data with each other. In the cache-per-host, the pilot jobs on a WN can discover and share cache data with each other. For $\mathrm{C} 1$ and $\mathrm{C} 2$, waitForData logic (as discussed in Section IV-E) is active in task scheduling process. In the following figures except Fig. 12, the $\mathrm{C} 1$ cache scheme has been used.

In order to study the effect in workflow latency in job submission and scheduling, three different job submission mechanisms have been used in these experiments which are 1) direct submission (noTQ), 2) job submission with already running pilot jobs and 3) job submission by submitting the pilot jobs on demand using the PilotMonitor. In the first submission mechanism, the TaskQueue and the pilot jobs are not used. The jobs are submitted directly to the Grid using the gLite software. In the second submission mechanism, 120 predefined pilot jobs are already running before the new jobs are enqueued into the TaskQueue. In this case, the pilot jobs are ready to acquire new jobs and execute them. In the third submission mechanism, the pilot jobs are submitted on demand using the PilotMonitor algorithm explained in Section IV-A.

The following paragraphs detail the results of the experiments that have been performed using the experimental setup discussed in the previous paragraphs. In order to measure these results, simulated experiments have been repeated several times and the figures present the measured average values and the standard deviation of the mean is shown as error bars. The plot in Fig. 7 shows the number of running jobs over time for a W3 workflow where the jobs were submitted using the three submission mechanisms. There is an initial job submission delay for the direct (without the pilot jobs and the TaskQueue) and PilotMonitor-based job submission. This delay is due to the scheduling latencies introduced by gLite, and pilot jobs have to wait in a local scheduler's queue before they can run and request the jobs. However, this is not the case when the pilot jobs are already running, and thus there are no submission delays as the pilots are already waiting for the jobs. There are no queuing delays for the jobs since the pilots pull them as far as they can to meet the jobs' requirements.

The results show a decrease in queuing times for the jobs and scheduling latencies when a pilot-based system is used in comparison to the direct submission. In the direct submission mechanism, there is also a huge delay between the time step0 jobs complete their execution and the jobs in step1 are submitted (group of running jobs as shown on the right side of the plot). This is due to the latency introduced by gLite in notifying the job completion. On the contrary, there are almost no such delays between these steps with the pilot-based approach. The knees in Fig. 7, for the pilot-based approach, are due to the delays in the submission of step1 jobs after the jobs in step0 have been completed. Fig. 7 shows how job submission, scheduling and job notification delays can be reduced using the pilot-based approach. The reduction in these delays is more than $60 \%$ for the PilotMonitor-based job submission and it is more than $75 \%$ when pre-allocated pilots have been used.

Fig. 8 shows the effect of stage-in delays on job execution times. The stage-in time in these experiments is the time a job spends in accessing and reading a file from an SE for processing. In CMS, files are read and processed directly from the SE and these files are not downloaded to a worker node. The plot shows that the system with the pilot cache provides much better data access times, especially under worse SE conditions (d3f3) as a smaller number of requests are sent to the SE, with an increasing number of datasets being available in the local caches. In the normal SE condition (d1f1), the cache does not offer significant advantage (less than $1 \%$ improvement) over the no-cache approach as the SE has low latencies and can serve the data requests almost as fast as is expected from the pilot cache. There is a minimal effect of higher delays and higher failure rates, i.e., $\mathrm{d} 2 \mathrm{f} 2, \mathrm{~d} 3 \mathrm{f} 1$, and $\mathrm{d} 3 \mathrm{f} 3$ on the stage-in time for the cache-based system because only a few files are read from the SE (from 10 to 13 seconds). On the contrary, with an increase in the delay and the failure rate, the stage-in time significantly increases (from 10 to 450 seconds) for the no-cache approach because all the files are read from the SE. It is important to note here that by using the cache, I/O requests to $\mathrm{SE}$ are decreased; therefore, its use may also minimize the deterioration of the SE conditions in the first place.

From Fig. 8, it is clear that the pilot cache mechanism positively affects the execution time of a job by reducing the data access latencies. Considering the mean deviations, the average reduction in job execution time is in the range $17 \%$ to $18 \%$ for $\mathrm{d} 3 \mathrm{f} 1$ and $18 \%$ to $20 \%$ for $\mathrm{d} 3 \mathrm{f} 3$ case. Since the jobs are inter-dependent in a workflow, this result should also reduce the turnaround time of a complete workflow as shown in Fig. 9.

From this figure, it is clear that, except for d1f1, the cache approach provides better workflow turnaround time than the no-cache approach. The average reduction in turnaround time is $17 \%$ to $20 \%$ for $\mathrm{d} 3 \mathrm{f} 1$ and $16 \%$ to $27 \%$ for $\mathrm{d} 3 \mathrm{f} 3$. An interesting fact to note here is that an increase in the failure rate has a more prominent effect on the turnaround time compared to an increase in the delay factor. This is due to the fact that a failure 


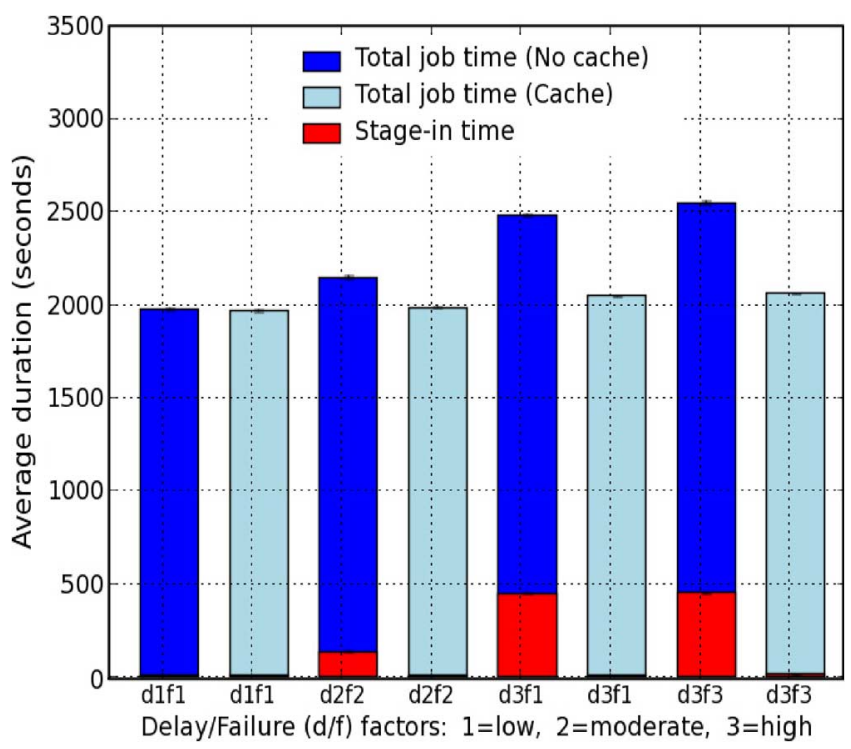

Fig. 8. Average job execution time and stage-in time within each job of W1 workflow using data caching and no-caching under different SE access conditions.

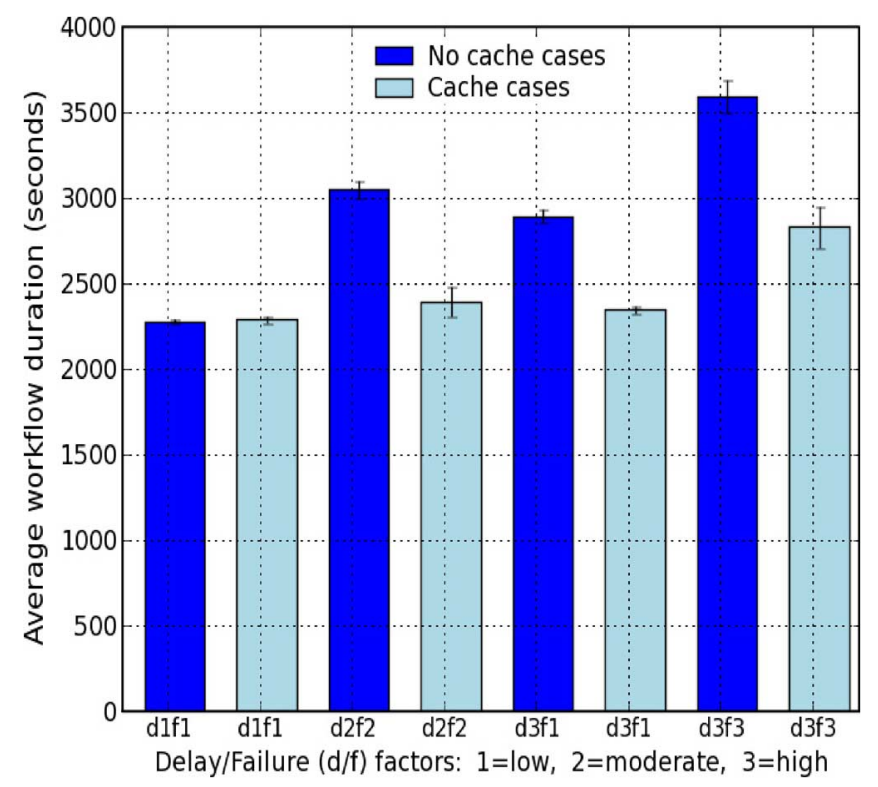

Fig. 9. Workflow turnaround time of W1 workflow using data caching and no-caching under different SE access conditions.

in reading or writing data to an SE causes a job to fail which triggers the resubmission of a job, and causes an additional delay of resubmission and re-execution of a job. We know that a job, in a workflow, cannot be ready for execution until its predecessor job has been completed. Since the failure of one job delays the start of its dependent job, it increases the turnaround time of a workflow.

In the case of the cache-based approach for W1, jobs mostly read the required files from the cache which reduces the data access latencies and the failures during the stage-in time. However, the failures at stage-out (writing data back to an SE) can lead to long workflow turnaround times. As a result, the turnaround time of $\mathrm{W} 1$ under $\mathrm{d} 3 \mathrm{f} 1$ (highest delay, low failure rate)

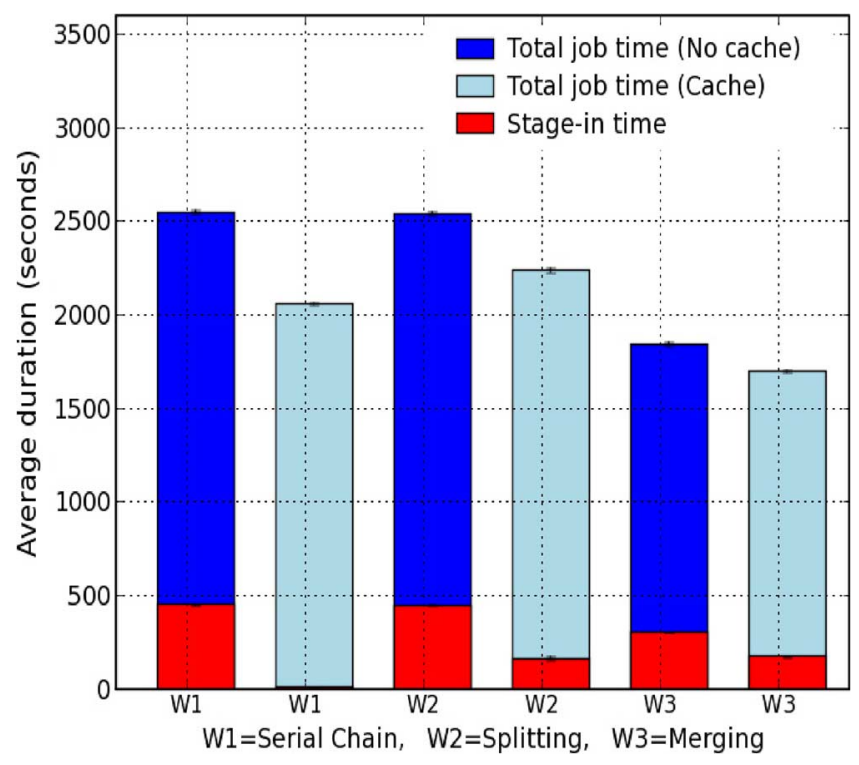

Fig. 10. Average job execution time and stage-in time of each job using data caching and no-caching for different types of workflow executed under high load conditions (d3f3) at SE.

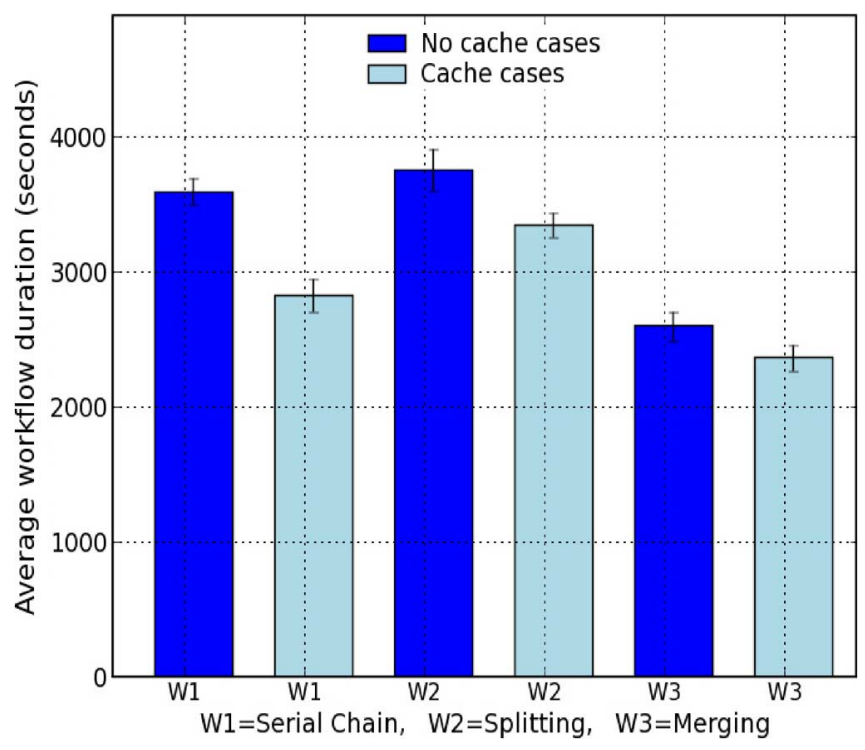

Fig. 11. Workflow turnaround times of different workflows using data cache and no-cache under high load conditions (d3f3) at SE.

condition for both the approaches, the cache and the no-cache, is less than $\mathrm{d} 2 \mathrm{f} 2$ and $\mathrm{d} 3 \mathrm{f} 3$.

Figs. 10 and 11 show the same behavior as discussed in Figs. 8 and 9 but with different workflows under worse SE conditions (d3f3). We can see that the cache mechanism performs much better ( $18 \%$ to $20 \%$ time reduction) for a workflow where the jobs show one-to-one dependency, i.e., the W1 workflow, because the jobs from step1 can be efficiently scheduled to the pilot jobs that hold the results of the jobs from step0.

For the workflows $\mathrm{W} 2$ and $\mathrm{W} 3$, the cache hit rate is less than the one in $\mathrm{W} 1$ because the jobs from step1 may be forced to read from two different pilots (in case of W3) or two jobs in different pilots may read from a single pilot that is holding the data (in case of W2). On average, for all the three workflows, the 


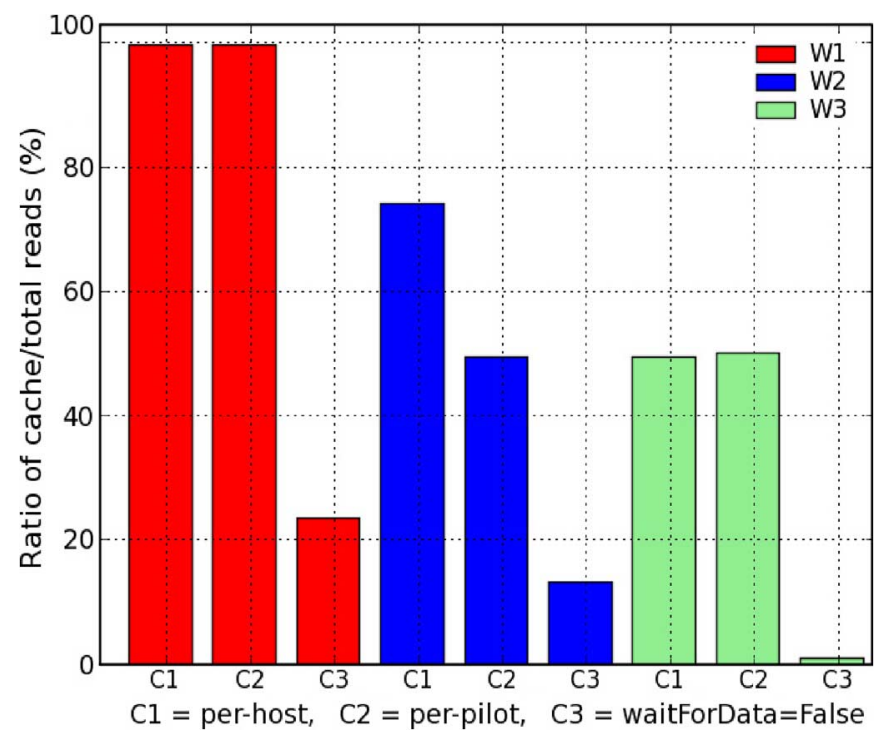

Fig. 12. Cache hit ratio for different cache configurations and workflow types.

system with the pilot cache behaves better than the one without it. The cache system shows $11 \%$ to $13 \%$ reduction in workflow turnaround time for W2, and $7 \%$ to $9 \%$ for W3. However, the cache hits can be further increased in case of $\mathrm{W} 2$ and $\mathrm{W} 3$ if a system with a global cache is used. The global cache means that the pilot jobs can share their data across WNs in a site.

Fig. 11 depicts the cache impact on the turnaround times of different workflows under worse SE conditions. Even if there is a greater dispersion of measurements in the case of $\mathrm{d} 3 \mathrm{f} 3$, it seems safe to say that the pilots with data cache help in improving the workflow execution time when the storage resources are in the stressed conditions. The reduction in the workflow turnaround time is $16 \%$ to $27 \%$ for $\mathrm{W} 1,4 \%$ to $17 \%$ for $\mathrm{W} 2$, and $1 \%$ to $16 \%$ for $\mathrm{W} 3$.

In this paper, we have discussed the single pilot cache (perpilot cache) and the cache sharing (cache-per-host) among the pilot jobs on a worker node. A caching scheme is measured on the basis of its responsiveness to the data access needs, mostly measured in terms of the cache hit ratio and the byte ratio. The cache hit ratio is the percentage of the data accesses that were found in the cache.

The illustration in Fig. 12 depicts the cache hit ratio for different caching combinations and different types of workflows. For the serial chain workflow (W1), the single pilot cache and the share cache with the waitForData policy, as explained in Section IV-E, yield hit rates above $99 \%$ because the jobs have a one-to-one data dependency and they are scheduled to those pilot jobs that are holding the required files in their caches. When the waitForData policy is not in use, the cache hit rate is severely reduced to $23 \%$ because the TaskQueue does not wait for the pilot jobs with the required data to request the job. Consequently, a job is scheduled to a pilot job that may not be holding the required files in its cache; thus, it may reduce the cache hit ratio. In the absence of a global cache, the waitForData approach appears to be fundamental to achieve a good cache hit ratio because it emphasizes more on data availability in the job scheduling process to increase the probability of cache hit.
In Fig. 12, we can see that the efficacy of the cache-per-host (C1) reaches to a $74 \%$ hit ratio for the splitting workflow (W2) whereas both cache-per-host and single pilot cache (C2) produce same $50 \%$ hit ratio for the merging workflow (W3). As we know, the step0 jobs can be scheduled to any pilot job because they do not have any data dependencies. Therefore, the outputs produced by these jobs are available randomly among all the pilot jobs. For W2, each step0 job produces two output files and each step1 job requires a single file as its input. In case of the single-pilot-cache, we may achieve $50 \%$ cache hits at the most. However, in case of the cache-per-host, the reason for a better cache hit ratio is due to the possibility that two jobs might be scheduled to two pilot jobs on the same $\mathrm{WN}$ where the required data was produced by a step0 job. Due to the cache sharing, the jobs can discover files available in some other pilot's cache, thus increasing the cache hit ratio.

In the case of W3, each job in step1 requires at least two input files produced by two different step0 jobs, which were executed by two different pilot jobs. It may be possible that those pilot jobs are running either on two different WNs or on the same WN. In any case, with the single-pilot-cache, $50 \%$ cache hits might be achievable. Since the pilots cannot share caches, each merge job can find at least one file from the pilot's cache. However, in the case of the cache-per-host, it might be possible that multiple required files are held by multiple pilot jobs running on the same WN (if the corresponding step0 jobs were executed on this WN). In this case, the scheduling mechanism may schedule the merge job to a pilot running on this WN. However, since the files produced by the step0 jobs are available randomly among the pilot jobs, the probability of finding two required files on the same WN is very low, and therefore, the cache hits ratio for the cache-per-host is only slightly higher than the single-pilot-cache for W3.

\section{CONCLUSIONS}

In this paper, we have proposed a pilot job with data cache approach to improve workflow scheduling and execution times for the CMS Tier0 analysis workflows. We have tested this framework using the Tier0 workflow on a dedicated setup at the CMS Tier0 infrastructure and at CIEMAT through simulated experiments. The workflow execution performed at Tier0 shows that the proposed system is able to successfully run a real CMS workflow, offering a small performance improvement in the case of a completely unloaded SE. We believe that the proposed caching strategy should be especially useful in cases of an overloaded SE and high data access times. In order to understand the efficacy of the proposed system, we devised the simulations where we can simulate the load on the SE.

First of all, using the pilot-based job submission, it has been seen that the delays incurred by the traditional gLite submission system can be reduced by a factor of $60 \%$ to $75 \%$. Further, the impact of data caching on the workflow execution time has been analyzed for three types of workflows. It has been noticed that cache provides negligible improvement in turnaround time if the $\mathrm{SE}$ is not overloaded. As mentioned earlier, the main use case of the data caching strategy is a scenario when the load on an SE is high, resulting in significant data access latencies and I/O errors. The caching strategy provides better results when such 
conditions are applied. It provides $27 \%$ reduction in turnaround time of a chain workflow, and provides $16 \%$ reduction in turnaround time of a merge workflow under worse SE conditions. The proposed caching strategy is helpful in protecting the SE from becoming overloaded by reducing load on them, and thus decreasing the need to over-provision these resources.

In order to improve the cache usability, shared per-host cache and a policy to reserve tasks for pilots holding required data (waitForData policy) have been introduced. Job scheduling algorithm using waitForData results in a $99 \%$ cache hit rate for the chaining workflow; however, it is reduced to $23 \%$ when waitForData policy is not applied. In the case of splitting workflow, the presence of per-host cache also helps improving the cache hit ratio: it rises from $50 \%$ to $74 \%$.

From these results, it can be concluded that pull-based job scheduling improves the turnaround time of a workflow by reducing the scheduling latencies faced by a job in Grid. It has also been observed that our caching strategy also contributes to improve the workflow turnaround time by reducing the data access latencies when the SE is under high load.

\section{REFERENCES}

[1] CMS Collaboration, The Compact Muon Solenoid Computing Technical Proposal, CERN/LHCC 1996-045, 1996.

[2] CMS Collaboration, The CMS Computing Model, CERN LHCC 2004035.

[3] F. Donno and M. Litmaath, Data Management in WLCG and EGEE, CERN-IT-Note-2008-002, Feb. 2008. [Online]. Available: http://cdsweb.cern.ch/record/1083660.

[4] M. Halem and R. Schauer, "A mass storage system administrator autonomic assistant," in Proc. 2nd Int. Conf. Autonomic Computing (ICAC), 2005, pp. 300-301.

[5] D. Lingrand, J. Montagnat, and T. Glatard, "Modeling the latency on production grids with respect to the execution context," in Proc. 8th IEEE Int. Symp. Cluster Computing and Grid (CCGRID), 2008, pp. 753-758.

[6] EGEE. [Online]. Available: http://www.eu-egee.org, visited Oct. 25, 2010

[7] G. Lo Presti and O. Barring et al., "CASTOR: A distributed storage resource facility for high performance data processing at CERN," in Proc. 24th IEEE Conf. Mass Storage Systems and Technologies (MSST), 2007, pp. 275-280.

[8] W. Allcock and I. Foster et al., "Globus toolkit support for distributed data-intensive science," in Proc. Computing in High Energy Physics (CHEP), Sep. 2001.

[9] M. U. Ahmed, R. A. Zaheer, and M. A. Qadir, "Intelligent cache management for data grid," in Proc. 2005 Australasian Workshop on Grid Computing and E-Research, 2005, pp. 5-12.

[10] F. Costa, L. Silva, I. Kelley, and I. Taylor, "Peer-to-peer techniques for data distribution in desktop grid computing platforms," in Proc. Core GRID Workshop Grid Programming Model, Grid \& P2P Systems Architecture, Grid Systems, Tools and Environment, Jun. 2007.
[11] B. Cohen, "Incentives build robustness in BitTorrent," in Proc. First Workshop Economics of Peer-to-Peer Systems, Jun. 2003.

[12] N. S. Good and A. Krekelberg, "Usability and privacy: A study of Kazaa P2P file-sharing," in Proc. SIGCHI Conf. Human Factors in Computing Systems, 2002, pp. 137-144.

[13] I. Taylor, A. Harrison, C. Mastroianni, and M. Shields, "Cache for workflows," in Proc. 2nd Workshop Workflows in Support of LargeScale Science, Jun. 2007, pp. 13-20.

[14] A. D. Peris, J. Hernández, E. Huedo, and I. Llorente, "Data location-aware job scheduling in the grid. Application to the Gridway metascheduler," in Proc. 17th Int. Conf. Computing in High Energy Physics (CHEP), 2009.

[15] T. Maeno et al., "Experience from a pilot based system for ATLAS," in Proc. Int. Conf. Computing in High Energy and Nuclear Physics (CHEP), 2007.

[16] S. K. Paterson and A. Tsaregorodtsev et al., "DIRAC optimized workload management," in Proc. Int. Conf. Computing in High Energy and Nuclear Physics (CHEP), 2007.

[17] S. Pablo et al., "AliEn: ALICE environment on the GRID," Nucl. Instrum. Meth. Phys., vol. 502, pp. 437-440, 2003.

[18] I. Sfiligoi, "glideinWMS-A generic pilot-based workload management system," in Proc. Int. Conf. Computing in High Energy and Nuclear Physics (CHEP07), 2007.

[19] M. Litzkow, M. Livny, and M. Mutka, "Condor-A hunter of idle workstations," in Proc. 8th Int. Conf. Distributed Computing Systems, Jun. 1988.

[20] Condor's Generic Connection Brokering. [Online]. Available: http:// www.cs.wisc.edu/condor/gcb/, visited Oct. 25, 2010.

[21] [Online]. Available: http://www.cs.wisc.edu/condor/manual/v7.4/ 3_7Networking_includes.html,visited Oct. 25, 2010.

[22] S. Shankar and D. Dewitt, "Data driven work-flow planning in cluster management systems," in Proc. 16th Int. Symp. High Performance Distributed Computing (HPDC), 2007, pp. 127-136.

[23] CMS Collaboration, The Computing Project Technical Design Report, CERN/LHCC-2005-023, 2005.

[24] F. Halzen and A. Martin, Quarks and Leptons: An Introductory Course in Modern Particle Physics. New York: Wiley, 1984.

[25] J. Hernández et al., "CMS Monte Carlo production in the WLCG computing grid," J. Phys., Conf. Series, vol. 119, 2008.

[26] E. Laura et al., Middleware for the Next Generation Grid Infrastructure (EGEE-PUB-2004-002), 2004.

[27] J. Frey, T. Tannenbaum, I. Foster, M. Livny, and S. Tuecke, "Condor-G: A computation management agent for multi-institutional grids," in Proc. 10th IEEE Symp. High Performance Distributed Computing (HPDC10), 2001.

[28] S. Zhou, "LSF: Load sharing in large-scale heterogeneous distributed systems," in Proc. Cluster Computing, Dec. 1992.

[29] M. Papakhian, "Comparing job management systems: The user's perspective," IEEE Computat. Sci. Eng., vol. 5, no. 2, pp. 4-9, Apr.-Jun. 1998.

[30] H. Li, D. Groep, L. Wolters, and J. Templon, "Job failure analysis and its implications in a large-scale production grid," in Proc. 2nd IEEE Int. Conf. eScience and Grid Computing, 2006.

[31] M. Arlitt and C. Williamson, "Trace-driven simulation of document caching strategies for internet web servers," Simulat. J., vol. 68, pp. 23-33, 1996.

[32] E. O'Neil, P. O'Neil, and G. Weikum, "The LRU-K page replacement algorithm for database disk buffering," in Proc. 1993 ACM SIGMOD Int. Conf. Management of Data, May 1993, pp. 297-306.

[33] J. Jeong and M. Dubois, "Cost-sensitive cache replacement algorithms," in Proc. 9th Int. Symp. High-Performance Computer Architecture (HPCA), 2003, pp. 327-337. 